\title{
Core Stability in Hedonic Games among Friends and Enemies: Impact of Neutrals
}

\author{
Kazunori Ota $^{1}$, Nathanaël Barrot ${ }^{1}$, Anisse Ismaili ${ }^{1}$, Yuko Sakurai ${ }^{2}$ and Makoto Yokoo ${ }^{1}$ \\ ${ }^{1}$ Kyushu University, Fukuoka 819-0395, Japan \\ ${ }^{2}$ National Institute of Advanced Industrial Science and Technology \\ nathanaelbarrot@gmail.com, anisse.ismaili@gmail.com, \\ ota@agent.inf.kyushu-u.ac.jp, yokoo@inf.kyushu-u.ac.jp, yuko.sakurai@aist.go.jp
}

\begin{abstract}
We investigate hedonic games under enemies aversion and friends appreciation, where every agent considers other agents as either a friend or an enemy. We extend these simple preferences by allowing each agent to also consider other agents to be neutral. Neutrals have no impact on her preference, as in a graphical hedonic game. Surprisingly, we discover that neutral agents do not simplify matters, but cause complexity. We prove that the core can be empty under enemies aversion and the strict core can be empty under friends appreciation. Furthermore, we show that under both preferences, deciding whether the strict core is nonempty, is $\mathrm{NP}^{\mathrm{NP}}$-complete. This complexity extends to the core under enemies aversion. We also show that under friends appreciation, we can always find a core stable coalition structure in polynomial time.
\end{abstract}

\section{Introduction}

Coalitions are a central part of economics, political, and social life. A natural question is whether a coalition structure (which is a partition of the set of agents) exists that is stable. In coalition formation games with hedonic preferences, each agent only cares about her own coalition. Since the number of coalitions that she can join is exponential, various compact classes of hedonic games have been proposed.

In particular, Dimitrov et al. [2006] developed games in which each agent divides the other agents into friends or enemies. They propose two alternative preferences. One is enemies aversion, where each agent prefers coalitions with fewer enemies and in case of a tie, with more friends. The other is friends appreciation, where each agent prefers coalitions with more friends and in case of a tie, with fewer enemies. Under enemies aversion, there always exists a core stable coalition structure that is NP-hard to find; and under friends appreciation, there always exists a strict core stable coalition structure, which can be found in polynomial-time.

In this paper, we examine a slight extension of this model where each agent divides the others into three groups, friends, enemies, and neutrals, who do not impact her preference, in the fashion of graphical hedonic games [Peters, 2016]. Indeed, in practice, agents commonly only care about a subset

\begin{tabular}{|c|c|c|}
\hline & Enemies aversion & Friends appreciation \\
\hline \multirow{3}{*}{ Core } & May be empty (Th. 1$)^{*}$ & Non-empty (Th. 9) ${ }^{*}$ \\
\hline & VERIF is coNP-c (Th. 5) & CONSTRUCTION takes \\
\hline & EXIST is NP ${ }^{N P}-c(T h .6)^{*}$ & polynomial time (Th. 9)* \\
\hline \multirow{3}{*}{$\begin{array}{l}\text { Strict } \\
\text { core }\end{array}$} & May be empty (Ex. 1) & May be empty (Th. 2) ${ }^{*}$ \\
\hline & VERIF is coNP-c (Th. 3)* & VERIF is coNP-c (Th. 7)* \\
\hline & EXIST is NP ${ }^{N P}-c(\text { Th. } 4)^{*}$ & EXIST is $\mathrm{NP}^{\mathrm{NP}}-\mathrm{c}(\mathrm{Th} .8)^{*}$ \\
\hline
\end{tabular}

Table 1: Summary of results: new ones marked with *.

of other agents; the rest are neutral. One might think that adding such a graphical assumption would simplify the computational problems or that since neutral agents do not impact preferences, the previous results in Dimitrov et al. [2006] would still hold in this extended model.

It turns out that under enemies aversion, a core stable coalition structure might not exist, and it might not exist under friends appreciation for the strict core either. Then, we investigate the complexity of (VERIF) to verify whether a given coalition structure is (strict) core stable and (EXIST) whether the (strict) core is non-empty. Our findings are in Table 1.

Related work Lang et al. [2015] proposed friendsneutrals-enemies hedonic games using the generalized Bossong-Schweigert extension principle. Peters [2016] considered graphical hedonic games. If the agent graph has a bounded treewidth and a bounded degree, deciding the core's existence is polynomial-time tractable. Aziz and Brandl [2012] clarified the inclusions between stability concepts such as core and Nash stability, and provided some existence results. Aziz et al. [2014] proposed conditions that guarantee a core stable outcome in fractional hedonic games. Aziz et al. [2016] proposed Boolean hedonic games where an agent partitions a set of other agents into satisfactory and unsatisfactory groups and showed core non-emptiness. Sung and Dimitrov [2007] showed that the verification problem for the core is coNP-complete in additive hedonic games. Woeginger [2013] showed that the existence problem for the core is $\mathrm{NP}^{\mathrm{NP}}$-complete in additively separable hedonic games (ASHG). Peters [2015] proved that the existence problem for the strict core is $\mathrm{NP}^{\mathrm{NP}}$-complete in ASHGs. Rey et al. [2016] show that under enemy-aversion, deciding the existence of the strict-core is DP-hard. Peters and Elkind [2015] developed a framework to prove the NP-hardness of existence problems, which widely applies to various hedonic games such as individually rational coalition lists [Ballester, 2004] and hedonic coalition nets [Elkind and Wooldridge, 2009]. 


\section{Model}

Let $N=\{1, \ldots, n\}$ denote the set of agents. A coalition $C \subseteq N$ is a subset of agents. A coalition structure $\pi$ is a partition of set $N$. Let $\pi(i)$ denote the coalition to which agent $i$ belongs in $\pi$. Let $C^{N}$ denote the set of coalition structures. For every agent $i$, her preference $\succsim_{i}$ is based on the coalitions to which she belongs; let $\succ_{i}$ (respectively $\sim_{i}$ ) denote the preference's asymmetric (respectively symmetric) part. A hedonic game $(N, P)$ is defined by set of agents $N$ and preference profile $P=\left(\succsim_{i}\right)_{i \in N}$.

Coalition structure $\pi \in C^{N}$ admits blocking coalition $\emptyset \neq X \subseteq N$ iff for every $i \in X, X \succ_{i} \pi(i)$ holds. The core is the set of coalition structures that do not admit any blocking coalition. Similarly, coalition structure $\pi \in C^{N}$ admits weakly blocking coalition $X \subseteq N$ iff for every $i \in X$, $X \succsim_{i} \pi(i)$, and there exists $j \in X$ such that $X \succ_{j} \pi(j)$. The strict core is the set of coalition structures that do not admit any weakly blocking coalition. ${ }^{1}$ Coalition $C$ is acceptable for agent $i$ if and only if $C \succsim_{i}\{i\}$ holds. $^{2}$

In this paper, we consider the following simple (and compact) preferences. For each agent $i$, set $N$ is partitioned into $\left\{F_{i}, E_{i}, \perp_{i}\right\}$. Agents $F_{i}$ are her friends, $E_{i}$ are her enemies, and $\perp_{i}$ are neutral agents $\left(i \in \perp_{i}\right)$.

Under enemies aversion, she first compares the number of enemies. Hence, without loss of generality, any coalition that contains an enemy is unacceptable. Within acceptable coalitions, she prefers coalitions with more friends, i.e., for two acceptable coalitions, $C \succ_{i} D$ holds iff $\left|C \cap F_{i}\right|>\left|D \cap F_{i}\right|$.

Under friends appreciation, she prefers coalitions with more friends, and in case of a tie, she prefers the one with fewer enemies: $C \succ_{i} D$ holds iff (a) $\left|C \cap F_{i}\right|>\left|D \cap F_{i}\right|$, or (b) $\left|C \cap F_{i}\right|=\left|D \cap F_{i}\right|$ and $\left|C \cap E_{i}\right|<\left|D \cap E_{i}\right|$.

In both preferences, $C \sim_{i} D$ holds iff $\left|C \cap E_{i}\right|=\left|D \cap E_{i}\right|$ and $\left|C \cap F_{i}\right|=\left|D \cap F_{i}\right|$. The set of preference profiles under enemies aversion (respectively friends appreciation) is denoted by $\mathcal{P}^{E}$ (respectively by $\mathcal{P}^{F}$ ). In $\mathcal{P}^{E}$ there is additive separability on weights $\{1,0,-n\}$, and in $\mathcal{P}^{F}$ on $\{n, 0,-1\}$. Definition 1 (HG/E and HG/F). An $H G / E$ is a hedonic game $\left(N,\left(\succsim_{i}\right)_{i \in N}\right)$ such that each $\succsim_{i}$ is in $\mathcal{P}^{E}$. Similarly, an $H G / F$ is a hedonic game $\left(N,\left(\succsim_{i}\right)_{i \in N}\right)$ such that each $\succsim_{i}$ is in $\mathcal{P}^{F}$.

Such hedonic games can be represented by a labeled digraph $G_{E F \perp}=\left(N, A_{E} \cup A_{F} \cup A_{\perp}\right)$ where each vertex represents an agent, and arc $(i, j)$ in set $A_{E}$ labeled by $E$ (respectively by $F, \perp$ ) indicates that for agent $i$, agent $j$ is an enemy (respectively friend, neutral). ${ }^{3}$

Example 1. [Dimitrov et al., 2006] Under enemies aversion, in this three-agent $\mathrm{HG} / \mathrm{E}$, the following preferences hold:

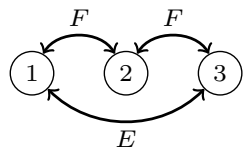

$$
\begin{gathered}
\{1,2\} \succ_{1}\{1\} \\
\{1,2,3\} \succ_{2}\{1,2\} \sim_{2}\{2,3\} \succ_{2}\{2\} \\
\{2,3\} \succ_{3}\{3\} .
\end{gathered}
$$

The coalition structures that do not admit a blocking coalition, are set $\{\{\{1,2\},\{3\}\}, \quad\{\{1\},\{2,3\}\}\}$. However, there are weak deviations from these coalition structures, respectively $\{2,3\}$ and $\{1,2\}$, and thus, the strict core is empty.

\footnotetext{
${ }^{1}$ The strict core is more demanding, thus contained in the core.

${ }^{2}$ If $\pi(i)$ is unacceptable, $\pi$ cannot be a member of the core.

${ }^{3}$ The size of this representation is $\Theta\left(n^{2}\right)$.
}

Depending on the context, we sometimes omit one of the three sets $A_{E}, A_{F}$ or $A_{\perp}$, since it can be deduced from the two others. For instance, omitting set $A_{\perp}$, graph $G_{E F}=$ $\left(N, A_{E} \cup A_{F}\right)$ shows a graphical hedonic game [Peters, 2016]. The following remark is also useful:

Remark 1. Under enemies aversion, consider an HG/E and graph $G_{F \perp}=\left(N, A_{F} \cup A_{\perp}\right)$, where only friendly and neutral arcs are represented, and enemy arcs omitted. Given coalition $C$, if arc $(i, j)$ is missing in subgraph $G_{F \perp}[C]$, then agent $j$ is an enemy of $i$, and $C$ is unacceptable to her. Thus, (i) if $\pi$ is a member of the (strict) core, for each $C \in \pi$, subgraph $G_{F \perp}[C]$ is necessarily a clique ${ }^{4}$, and (ii) given $\pi$ where every coalition $C \in \pi$ induces clique $G_{F \perp}[C]$, a (weakly) blocking coalition $X \subseteq N$ necessarily induces clique $G_{F \perp}[X]$.

\section{Stable Coalitions May Not Exist.}

In this section, we discuss the existence of a (strict) core stable coalition structure. Under enemies aversion, considering only friends and enemies (no neutrals), the possible nonexistence of a strict core stable coalition structure was shown in Example 1 [Dimitrov et al., 2006] and implies that the strict core can be empty, when neutral agents are also allowed.

This example does not extend to the (non strict) core in the friends-and-enemies model and indeed a core stable coalition structure always exists under enemies aversion [Dimitrov et al., 2006]. However, when we add neutral agents to the friends-and-enemies model, the following theorem holds.

Theorem 1. In an $H G / E$, the core can be empty.

To prove this theorem, we utilize the following example. Example 2. Assume 15 agents who are divided into a cycle of five groups, $C_{0}, \ldots, C_{4}$, each of which contains three agents. $C_{i+k}$ (resp. $C_{i-k}$ ) denotes $C_{i+k \bmod 5}\left(\right.$ resp. $C_{i-k \bmod 5}$ ). The sets of friends, neutrals and enemies are as follows:

- Agents in the same group are friends.

- Agents in $C_{j}$ consider agents in $C_{j \pm 2}$ enemies.

- Agents in $C_{j}$ consider agents in $C_{j+1}$ friends.

- Agents in $C_{j}$ consider one agent in $C_{j-1}$ a neutral and the other two agents to be friends.

We illustrate these preferences with a partial representation of graph $G_{F \perp}$ by focusing on group $C_{0}$ :
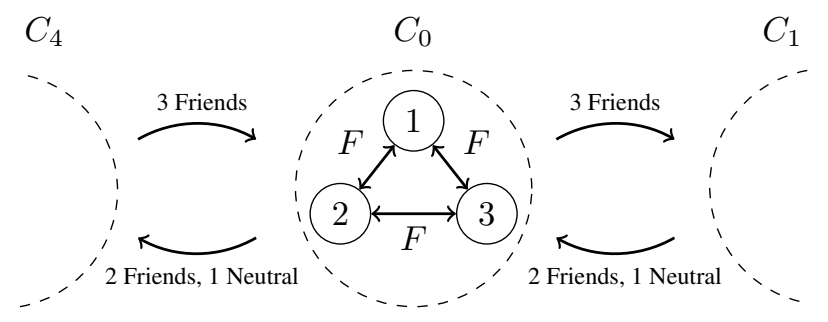

Proof of Theorem 1. Assume $\pi$ is core stable in Example 2. From Remark 1, only the agents in adjoining groups can form a coalition. Also, note that if coalition $C_{i} \cup C_{i+1}$ is formed, each member in $C_{i}$ is with 5 friends and each member in $C_{i+1}$ is with 4 friends. First, we show that the agents in a same

${ }^{4}$ A digraph $G=(W, B)$ is a clique iff $\forall(i, j) \in W^{2},(i, j) \in B$. 
group cannot be separated. For the sake of contradiction, assume agent $i \in C_{0}$ is separated from the other members in $C_{0}$. There are three cases to study:

- When $\pi(i) \cap C_{1} \neq \emptyset: i$ is with at most 3 friends, and $j \in C_{0}(j \neq i)$ is with at most 3 friends. Each member in $C_{4}$ is also with at most 4 friends (since they are not with $i$ ). Then $C_{4} \cup C_{0}$ is a deviation.

- When $\pi(i) \cap C_{4} \neq \emptyset$ : each member of $C_{4}$ is with at most 3 friends. Also, each member of $C_{3}$ is with at most 4 friends. Then, $C_{3} \cup C_{4}$ is a deviation.

- When $\pi(i)=\{i\}$ : the only case for which $C_{0} \cup C_{1}$ (or $\left.C_{0} \cup C_{4}\right)$ is not a deviation is when $\left(C_{0} \backslash\{i\}\right) \cup C_{1}$ forms a coalition (such that at least one member in $C_{1}$ does not strictly prefer $C_{0} \cup C_{1}$ ). Here, each member in $C_{1}$ is with at most 4 friends. Then, consider the members of $C_{2}$. The only way that a member of $C_{2}$ is with 4 or more than 4 friends is to form a coalition with 2 or 3 members of $C_{3}$. However, in such a case, each member of $C_{3}$ is with at most 4 friends and each member in $C_{4}$ is with at most 3 friends, and therefore, $C_{3} \cup C_{4}$ becomes a deviation. Thus, each member in $C_{2}$ is with at most 3 friends, but then $C_{1} \cup C_{2}$ is a deviation.

Using a similar argument, we can prove that all the members in the same group must be in the same coalition in $\pi$. Also, it is not possible that two adjoining groups, $C_{j}$ and $C_{j+1}$, are isolated, i.e., $C_{j} \in \pi$ and $C_{j+1} \in \pi$, since $C_{j} \cup C_{j+1}$ becomes a deviation. Therefore, consider coalition structure $\pi$ where no two consecutive groups are alone, which implies that exactly one $C_{j}$ is alone. Without loss of generality, we assume that just $C_{0}$ is alone. Then the only coalition structure that is not discarded by Remark 1 is $\pi=\left\{C_{0}, C_{1} \cup C_{2}, C_{3} \cup C_{4}\right\}$. Then each member in $C_{0}$ is with 2 friends and each member in $C_{4}$ is with 4 friends, and thus, $C_{4} \cup C_{0}$ is a deviation. Therefore, no core stable coalition structure exists.

Under friends appreciation and considering only friends and enemies, both the core and the strict core always exist [Dimitrov et al., 2006]. However, if we add neutral agents, then we have the following:

Theorem 2. In an $H G / F$, the strict core can be empty.

To prove this theorem, we utilize the following example. Example 3. Consider $N=\{1,2,3\}$ under friends appreciation based on partitions $F_{1}=\{1,2\}, E_{1}=\{3\}, F_{2}=\{2\}$, $\perp_{2}=\{1,3\}, F_{3}=\{2,3\}$ and $E_{3}=\{1\}$.

Proof of Theorem 2. The core contains all of the coalition structures except $\{\{2\},\{1,3\}\}$. However, there is a weak deviation from all coalition structures in the core, either $\{1,2\}$ or $\{2,3\}$, and thus, the strict core is empty.

\section{Problems and Complexity}

The previous section showed that in an $\mathrm{HG} / \mathrm{E}$, both the strict core and the core may be empty, and in an $\mathrm{HG} / \mathrm{F}$, the strict core may be empty. These observations bring to surface the following decision problems to decide the non-emptiness of the strict core and the core for an $\mathrm{HG} / \mathrm{E}$ as well as the non-emptiness of the strict core for an
HG/E: (i) HG/E/SC/ExIST, (ii) HG/E/C/ExIST, and (iii) $\mathrm{HG} / \mathrm{F} / \mathrm{SC} / \mathrm{ExIST}$. To examine whether they belong to class $\mathrm{NP}$, we also study the problem of verifying whether a given coalition structure $\pi$ is in the core or the strict core for a given game: i.e., (a) HG/E/SC/VERIF, (b) HG/E/C/VERIF, and (c) $\mathrm{HG} / \mathrm{F} / \mathrm{SC} / \mathrm{VERIF}$. We address the complexity of these problems in the next sections.

Class NP corresponds to the set of decision problems where 'yes'-instances allow a polynomially-sized certificate verifiable in polynomial-time. For instance, in a further proof, we utilize problem MAXCLIQUE, which is among the computationally most intractable problems in class NP. Indeed, it is NP-complete: it is in NP and NP-hard. ${ }^{5}$

Definition 2 (Problem MAXCLIQUe). Given graph $\mathcal{G}=$ $(\mathcal{V}, \mathcal{A})$ and lower threshold $k \in \mathbb{N}$, does a subset of $k$ vertices $\mathcal{W} \subseteq \mathcal{V}$ exist such that subgraph $\mathcal{G}[\mathcal{W}]$ is a clique?

Complementation consists in transposing the 'yes' and 'no' answers. Consequently, class coNP is symmetric to class $\mathrm{NP}$ and corresponds to the set of decision problems where 'no'-instances allow a polynomially-sized certificate verifiable in polynomial-time. For instance, the problem of verifying that a given coalition structure is core stable belongs to class coNP, since we can certify 'no'-instances with a blocking coalition. One can show that a problem is coNP-complete by proving that it is in coNP and that it is the complement of an NP-hard problem, by symmetry of NP and coNP.

A decision problem may also neither allow a yes or a no verification in polynomial-time, falling outside of NP and coNP. Class $N P^{N P}$ corresponds $^{6}$ to the decision problems for which 'yes'-instances allow a polynomially-sized certificate that is verifiable in polynomial-time using a constant-time NP-oracle. ${ }^{7}$ Class coNP ${ }^{N P}$ is its complement. ${ }^{8}$ Typically, a problem in $\mathrm{NP}^{\mathrm{NP}}$ is extremely hard, since it consists in a coNP problem nested into an NP problem. Problem MINMAXCLIQUE is typical for this second level of the polynomial hierarchy.

Definition 3 (Problem MinMaxCliQue). Given graph $\mathcal{G}=$ $(\mathcal{V}, \mathcal{A})$, two sets $I, J$ that partition set $\mathcal{V}$ into $\left\{\mathcal{V}_{i, j} \mid i \in I, j \in\right.$ $J\}$, and lower threshold $k \in \mathbb{N}$, does, for every function $t$ : $I \rightarrow J$, subgraph $\mathcal{G}\left[\cup_{i \in I} \mathcal{V}_{i, t(i)}\right]$, contain a $k$-sized clique?

Intuitively, $\mathcal{V}$ is partitioned into $|I| \cdot|J|$ subsets $\mathcal{V}_{i, j}$. According to function $t,|I|$ subsets are chosen (for each $i \in I$, set $\left.\mathcal{V}_{i, t(i)}\right)$. Then for the union of these subsets, we consider MaxCliQue. There are $|J|^{|I|}$ variations of function $t$. Problem MinMaxCliQue lies in class coNP ${ }^{\mathrm{NP}}$, since by inferring the correct function $t: I \rightarrow J$ (a 'no' certificate), the NPoracle can solve the MAXCLIQUE problem on $\mathcal{G}\left[\cup_{i \in I} \mathcal{V}_{i, t(i)}\right]$ and verify the 'no' answer. Completeness is defined in a standard manner with polynomial-time reductions, and we know:

Lemma 1. [Ko and Lin, 1995] Problem MINMAXCLIQUE is coNP $P^{N P}$-complete. (Their proof even holds when $J=\{0,1\}$ and $\left|\mathcal{V}_{i, 0}\right|=\left|\mathcal{V}_{i, 1}\right|$ for every $i \in I$.)

\footnotetext{
${ }^{5}$ Any problem from class NP can be reduced to MAXCLIQUE in polynomial-time; so that solving it efficiently would solve P vs NP.

${ }^{6}$ Class $\Sigma_{2}^{P}$ in the second level of the Polynomial Hierarchy.

${ }^{7} \mathrm{~A}$ blackbox that solves any (co)NP problem in constant-time.

${ }^{8}$ Class $\Pi_{2}^{P}$ in the second level of the Polynomial Hierarchy.
} 


\section{Enemies Aversion and the Strict Core}

In this section, under enemies aversion, we first address the complexity of verifying that a given coalition structure is in the strict core. Second, we address the complexity of deciding whether the strict core is non-empty. We show the following:

\section{Theorem 3. Problem HG/E/SC/VERIF is coNP-complete.}

Hence the existence problem does not allow a classical verification procedure in polynomial-time. Indeed, it is not in NP or coNP, since we show that:

Theorem 4. Problem HG/E/SC/EXIST is $N P^{N P}$-complete.

Therefore, since this problem is at least as intractable as all the problems that nest a coNP problem into an NP problem, it is extremely intractable, despite the utter simplicity of enemies aversion as a preference.

\subsection{Proving Complexity of Verification}

Proof of Theorem 3. Problem HG/E/SC/VERIF is in coNP, since 'no'-instances can be certified with a weakly blocking coalition. For coNP-hardness, we reduce problem MAXCLIQUE to the complement of problem HG/E/SC/VERIF.

Let graph $\mathcal{G}=(\mathcal{V}, \mathcal{A})$ and threshold $k \in \mathbb{N}$ define an instance of MAXCLIQUE. From it, we construct an HG/E/SC/VERIF instance with vertex-agents $V \equiv \mathcal{V}, k-1$ weight-agents in set $K$, and one fulcrum-agent $\varphi$; therefore with set of agents $N=V \cup\{\varphi\} \cup K$. Bearing Remark 1 in mind, we depict graph $G_{F \perp}$ in Fig. 1, and all other arcs are enemies. Concerning set $V$, for every edge $\{i, j\}$ in graph $\mathcal{G}=(\mathcal{V}, \mathcal{A})$ we construct neutral $\operatorname{arcs}(i, j)$ and $(j, i)$. In set $K$, all $k-1$ agents are mutual friends. Between sets $V$ and $K$, fulcrum-agent $\varphi$ shares a mutual friendship with everyone. Finally, in given coalition structure $\pi$, every vertexagent $i \in V$ is in singleton $\{i\}$, and the fulcrum-agent forms a coalition with the $k-1$ weight agents.

In coalition structure $\pi$, there is a (weakly) blocking coalition if and only if the fulcrum-agent can improve (from $k-1$ to at least $k$ friends) with a $k$-sized clique in set $V$, and hence if and only if a $k$-sized clique exists in graph $\mathcal{G}$.

\subsection{Proving Complexity of Existence}

Proof of Theorem 4. Problem HG/E/SC/EXIST is in NP ${ }^{\mathrm{NP}}$. For 'yes'-instances, a coalition structure $\pi$ in the strict core is a certificate that can be verified using an NP-oracle. (Recall that the verification problem is coNP-complete.)

We prove that problem $\mathrm{HG} / \mathrm{E} / \mathrm{SC} / \mathrm{EXIST}$ is $\mathrm{NP}^{\mathrm{NP}}$-hard by showing that the coNP ${ }^{\mathrm{NP}}$-complete problem MINMAXCLIQUE (Lemma 1) can be reduced to the complement of problem HG/E/SC/EXIST. Let graph $\mathcal{G}=(\mathcal{V}, \mathcal{A})$, set $I$, which partitions set $\mathcal{V}$ into $\left\{\mathcal{V}_{i, 0}, \mathcal{V}_{i, 1} \mid i \in I\right\}$ and lower

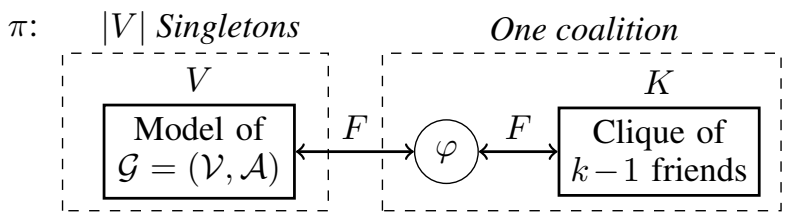

Figure 1: Reducing MAXCLIQUE to co-HG/E/SC/VERIF: The edges of graph $\mathcal{G}$ become neutral arcs for agents $V$. threshold $k \in \mathbb{N}$ define a restricted instance of MinMAXClique where $\forall i \in I,\left|\mathcal{V}_{i, 0}\right|=\left|\mathcal{V}_{i, 1}\right|$ holds. We construct an instance of coHG/E/SC/EXIST addressing the MINMAXCLIQUE instance, as follows. Graph $G_{F \perp}$ is partially shown in Fig. 2, by keeping Remark 1 on necessary cliques in mind.

(1) For every set of vertices $\mathcal{V}_{i, j}$, we introduce set of vertex-agents $V_{i, j} \equiv \mathcal{V}_{i, j}$. For every edge $\{x, y\}$ in graph $\mathcal{G}=(\mathcal{V}, \mathcal{A})$, we introduce neutral $\operatorname{arcs}(x, y)$ and $(y, x)$ between the agents of $V=\bigcup_{i \in I}\left(V_{i, 0} \cup V_{i, 1}\right)$. (There is no edge between $\mathcal{V}_{i, 0}$ and $\mathcal{V}_{i, 1}$.) (2) We introduce two friend-cliques $K_{0}$ and $K_{1}$, each of which contains $k-2$ mutual friends. Between them, agent $y$ is a mutual friend with everyone in $K_{0}$ and $K_{1}$. (3) Fulcrum-agent $\varphi$ is a mutual friend with agent $y$, with everyone in clique $K_{0}$ (but no one in clique $K_{1}$ ) and with everyone in set $V$. (4) Between every pair of sets $V_{i, 0}$ and $V_{i, 1}$, we introduce $\left|\mathcal{V}_{i, 0}\right|=\left|\mathcal{V}_{i, 1}\right|$ inhibitors (specified below) by pairing the agents of $V_{i, 0}$ and $V_{i, 1}$. Each inhibitor, which is a game connected to one vertex in $\mathcal{V}_{i, 0}$ and one in $\mathcal{V}_{i, 1}$, makes the former vertex xor the latter non-available. (5) To avoid inhibitors going to two different sides for one $V_{i, j}$, we connect to every set $V_{i, j}$ a circuit game $L_{i, j}$ (specified below) in which the strict core is non-empty if and only if the "every agent in $V_{i, j}$ is inhibited, or none is inhibited" condition is satisfied. (6) Other arcs are for enemies.

Recall that we want to show that our reduction addresses problem MinMaXCLIQUE. Therefore, we want to show:

$$
\begin{aligned}
& \forall t: I \rightarrow\{0,1\}, \exists k \text {-sized clique } \in \mathcal{G}\left[\cup_{i \in I} \mathcal{V}_{i, t(i)}\right] \\
\Leftrightarrow \quad & \forall \pi \in C^{N}, \exists \text { weakly blocking coalition } X \subseteq N
\end{aligned}
$$

But first, observe that in our construct, assuming strict core stability, fulcrum-agent $\varphi$ is either grouped with $y$ and $K_{0}$ ( $k-1$ friends) or with a clique in $V$ of at least $k$ friends. If agent $\varphi$ goes to a clique in $V$, then the game on agents $K_{1} \cup\{y\} \cup K_{0}$ is isolated with an empty strict core. (If $y$ groups with $K_{0}$, then coalition $\{y\} \cup K_{1}$ weakly blocks; and if $y$ groups with $K_{1}$, then coalition $\{y\} \cup K_{0}$ weakly blocks.) If agent $\varphi$ goes to the right, then the strict core of game $\{\varphi\} \cup K_{1} \cup\{y\} \cup K_{0}$ is non-empty: agents $\varphi, K_{0}, y$ group and agents $K_{1}$ group. We say that an agent is available for forming a coalition $C$ if she does not worse off.

('no' $\Rightarrow$ 'no'): Let function $t^{*}: I \rightarrow\{0,1\}$ be such that subgraph $\mathcal{G}\left[\cup_{i \in I} \mathcal{V}_{i, t^{*}(i)}\right]$ contains no clique of size $k$, and construct a coalition structure with no weakly blocking coalition. For every $i \in I$, we put all the inhibitors between $V_{i, 0}$ and $V_{i, 1}$ on $V_{i, 1-t^{*}(i)}$ (so that no circuit game $L$ generates a weakly blocking coalition). Hence, only agents

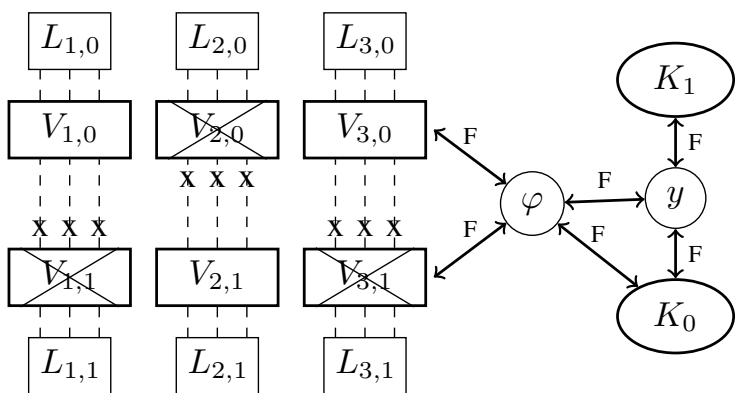

Figure 2: From MinMaxCLIQUE to coHG/E/SC/EXIST: The corresponding graph $G_{F \perp}$ specified above. 
$\cup_{i \in I} V_{i, t^{*}(i)}$ are available for grouping with fulcrum-agent $\varphi$, but never in a clique larger that $k-1$ (based on the premise). Therefore, by grouping agents $\varphi, y$, and $K_{0}$ into one coalition, fulcrum-agent $\varphi$ would worse off by deviating within $\cup_{i \in I} V_{i, t^{*}(i)}$. To conclude, by forming singletons with each agent in $\cup_{i \in I} V_{i, t^{*}(i)}$ and a coalition with agents $K_{1}$, this coalition structure admits no weakly blocking coalition.

('yes' $\Rightarrow$ 'yes'): Assume that for every function $t: I \rightarrow$ $\{0,1\}$, subgraph $\mathcal{G}\left[\cup_{i \in I} \mathcal{V}_{i, t(i)}\right]$ contains a clique of size $k$, and for the sake of contradiction let coalition structure $\pi$ admit no weakly blocking coalition. Then there exists $t^{(\pi)}$ such that for every $i \in I$, all the inhibitors between $V_{i, 0}$ and $V_{i, 1}$ go to side $1-t^{(\pi)}(i)$, or otherwise circuit games $L_{i, 0}$ and $L_{i, 1}$ contain a weakly blocking coalition. Consequently, fulcrumagent $\varphi$ is not grouped with agents $y$ and $K_{0}$, but at least with a $k$-sized clique in $G_{F \perp}\left[\cup_{i \in I} V_{i, t(\pi)}{ }_{(i)}\right]$ that exists based on the premise. Then the game on agents $K_{1} \cup\{y\} \cup K_{0}$ is isolated. However, its strict core is empty, a contradiction.

Inhibitors pair every vertex $x$ in set $V_{i, 0}$ with a vertex $y$ in $V_{i, 1}$. Their construction is depicted in graph $G_{F \perp}$ below.

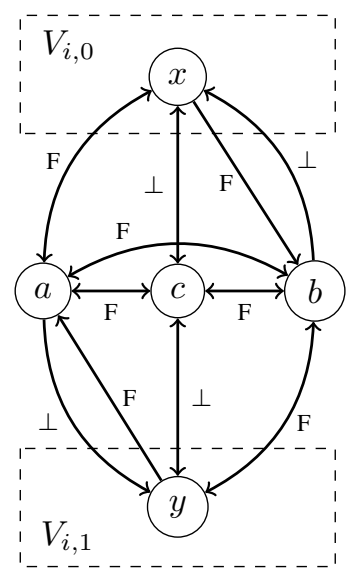

Crucially, each inhibitor is an enemy of the other agents, preventing $x$ xor $y$ to participate in the game.

We show that inhibitor-clique $\{a, c, b\}$ is either grouped with agent $x$ xor agent $y$, and if it is grouped with agent $x$ (resp. $y$ ), then agent $x$ (resp. $y$ ) prefers to stay with the inhibitor upon every other coalition, since she has two friends in it.

If agent $c$ joins $x$, then $a$ and $b$ follow. Similarly, if $c$ joins $y$, then $b$ and $a$ follow. Finally, if $c$ is not grouped with $x$ or $y$, then $a$ and $b$ join $x$ and $y$, and $c$ is interested in joining $x$ or $y$, and $a$ and $b$ follow $c$.

Each circuit game $L_{i, j}$ is connected to the agents of set $V_{i, j}$ and constructed so that its strict core is non-empty if and only if "every agent in $V_{i, j}$ is inhibited, or none is inhibited." This construction relies on a combination of smaller gadget games that model any logical gate under the interpretation where an agent available amounts to Boolean true. Gates NOT, OR, and DUPLIC with inputs $x$ and outputs $y$ (Fig 3) are sufficient to obtain a Boolean algebra. In gate NOT, the availability of $x$ makes $y$ non-available. In gate $\mathrm{OR}$, the availability of $x_{1}$ or $x_{2}$ makes $\alpha$ non-available and $y$ available. In gate DUPLIC, the availability of $x$ is duplicated into $y_{1}$ and $y_{2}$. Note that gate $\operatorname{AND}\left(x_{1}, x_{2}\right)$ equals $\operatorname{NOT}\left(\operatorname{OR}\left(\operatorname{NOT}\left(x_{1}\right), \operatorname{NOT}\left(x_{2}\right)\right)\right)$, and gates OR and AND generalize from binary operators to multinary ones.

By combining these gadget games, circuit game $L_{i, j}$ is constructed to obtain formula $\left(\bigwedge_{x \in V_{i, j}} x\right) \bigvee\left(\bigwedge_{x \in V_{i, j}} \neg x\right)$ as the following availability of output agent $y$ : "everyone or no-one" (Fig. 4). To ensure that the entire game (of the reduction) is not altered by the agents of the logic game, every agent $x$ in set $V_{i, j}$ is separated from $L_{i, j}$ by a (double negation) gate where agent $s$ is mutually neutral with every other
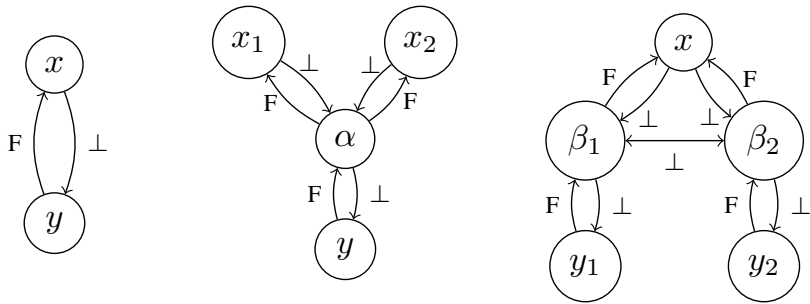

Figure 3: Gates NOT, OR and DUPLIC (inputs $x$, outputs $y$ )

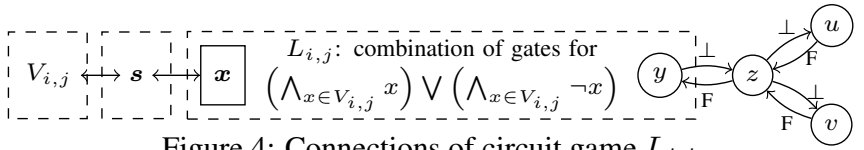

Figure 4: Connections of circuit game $L_{i, j}$

agent; when $x$ is available, both $x$ and $s$ can join the fulcrum.

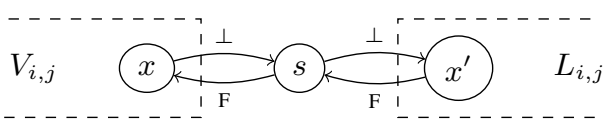

Finally, the validity of the formula and the availability of agent $y$ make $z$ non-available for $u$ and $v$ who remain stable singletons. Otherwise, the game on agents $\{u, z, v\}$ is isolated and has an empty strict core. To summarize, the circuit game has a non-empty strict core if and only if formula $\left(\wedge_{x \in V_{i, j}} x\right) \vee\left(\wedge_{x \in V_{i, j}} \neg x\right)$ holds.

\section{Extension to Enemies Aversion and the Core}

In this section, under enemies aversion, we extend the results on complexity of existence obtained for the strict core to the core. First, the complexity of verification was addressed in the proof of (Th.1) [Sung and Dimitrov, 2007]:

Theorem 5 ([Sung and Dimitrov, 2007], Proof of Th.1). Problem $\mathrm{HG} / \mathrm{E} / \mathrm{C} / \mathrm{VERIF}$ is coNP-complete.

There might be no polynomial-time verification procedure for the existence problem. Indeed, we show the following:

Theorem 6. Problem $\mathrm{HG} / \mathrm{E} / \mathrm{C} / \mathrm{EXIST}$ is $N P^{N P}$-complete.

Proof (sketch). First, problem HG/E/C/EXIST is in NP ${ }^{\mathrm{NP}}$, since, for 'yes'-instances, a coalition structure $\pi$ in the core is a certificate that can be verified easily using an NP-oracle.

As previously, we prove that $\mathrm{HG} / \mathrm{E} / \mathrm{C} / \mathrm{EXIST}$ is $\mathrm{NP}^{\mathrm{NP}}$ hard by showing that MINMAXCLIQUE can be reduced to coHG/E/C/EXIST. The proof follows the same ideas developed for the strict core, relying on inhibitors and circuit games. However, due to the structure of circuit games here, we need to introduce vertex-cliques (resp. a fulcrum-clique) instead of vertex-agents (resp. a fulcrum-agent), as follows:

(1) First, for each vertex $x$ in $\mathcal{V}$ we introduce a vertexclique, $K_{x}$, that contains $k^{\prime}$ mutual friends $\left(k^{\prime}\right.$ is specified below). For all edges $\{x, y\}$ in graph $\mathcal{G}=(\mathcal{V}, \mathcal{A})$, we introduce mutual neutral arcs between each $x^{\prime} \in K_{x}$ and $y^{\prime} \in K_{y}$. (2) Second, we introduce a generalization of Example 2 with five cliques $\left\{C_{0}, \ldots, C_{4}\right\}$, each of which contains $\left(k^{\prime \prime}-1\right)$ mutual friends ( $k^{\prime \prime}$ is specified below). (3) Third, we introduce a fulcrum-clique of $k^{\prime \prime}$ mutual friends, $K_{\varphi}$. Each agent in $K_{\varphi}$ is a mutual friend with each agent in $C_{0}$ and with 
Proceedings of the Twenty-Sixth International Joint Conference on Artificial Intelligence (IJCAI-17)
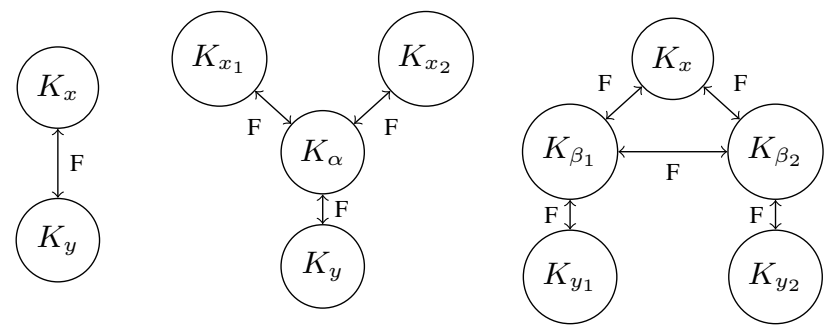

Figure 5: Gates NOT, OR and DUPLIC (in: $K_{x}$, out: $K_{y}$ )

everyone in set $V$. (4) Finally, inhibitors and circuit games (specified below) play the same role as for the strict core.

The main argument for this reduction's validity is the same as for the strict core setting. Fulcrum-clique $K_{\varphi}$ is either grouped with $C_{0}\left(\left(k^{\prime \prime}-1\right)\right.$ friends) or with at least a $k^{\prime \prime}$-sized clique in $V$, in a core stable coalition. Therefore, we only present the inhibitors and the circuit games.

An inhibitor is a clique that contains $\left(k^{\prime \prime}+1\right)$ mutual friends and pairs a vertex-clique $K_{x}$ in $V_{i, 0}$ with a vertexclique $K_{y}$ in $V_{i, 1}$. Each agent of the inhibitor-clique is a mutual friend with every agent in $K_{x}$ and $K_{y}$. Thus, the inhibitor-clique either groups with $K_{x}$ or $K_{y}$ in a core stable coalition structure. If the inhibitor-clique is grouped with $K_{x}$ (resp. $K_{y}$ ), then $K_{x}$ (resp. $K_{y}$ ) prefers to stay with the inhibitor upon every other coalition.

Each circuit game $L_{i, j}$ follows the same principle as in Theorem 4, relying on logic gates NOT, OR, and DUPLIC (Fig. 5) to obtain a Boolean algebra. However, in gate OR, we have to assume that $K_{x_{1}}$ or $K_{x_{2}}$ are two friends-cliques with identical size $t$, and we set the size of $K_{\alpha}$ and $K_{y}$ to $(t-1)$. Then the availability of $K_{x_{1}}$ or $K_{x_{2}}$ makes $K_{\alpha}$ nonavailable and $K_{y}$ available. In gate DUPLIC, assuming the size of $K_{x}$ is $t \geq 3$, we set the sizes of $K_{\beta_{1}}$ and $K_{\beta_{2}}$ to $(t-2)$, and the size of $K_{y_{1}}$ and $K_{y_{2}}$ to $(t-1)$. Then the availability of $K_{x}$ is duplicated into $K_{y_{1}}$ and $K_{y_{2}}$.

As in Theorem 4, using these gates, circuit game $L_{i, j}$ can be constructed, and we connect its output to a specific instance of Example 2 with five cliques of size 3. Also, between each vertex-clique $K_{x}$ and $L_{i, j}$, we introduce a double negation, composed of a separating-clique of size $k^{\prime}$ and a second clique of size $\left(k^{\prime}-1\right)$. To conclude, since the circuit game leads us to set $k^{\prime}=9$, we set $k^{\prime \prime}=18 k$ because the agents of the separating-clique have to be mutual friends with each agent of the fulcrum-clique to guarantee core stability.

\section{On Friends Appreciation}

In this section, we consider hedonic games under friends appreciation. Even though with only friends and enemies the existence of a strict core stable coalition structure is guaranteed, Theorem 2 shows that the existence is not guaranteed with neutral agents. Furthermore, we show the following:

Theorem 7. Problem HG/F/SC/VERIF is coNP-complete.

Proof (sketch). Surprisingly, the same reduction as for Theorem 3 works with a slight modification: vertex-agents are neutral toward the fulcrum. Then the preferences of vertexagents and the fulcrum lie in $\mathcal{P}^{E} \cap \mathcal{P}^{F}$. Moreover, the clique of friends cannot be grouped with vertex-agents.
Theorem 8. Problem $\mathrm{HG} / \mathrm{F} / \mathrm{SC} / \mathrm{EXIST}$ is $N P^{N P}$-complete.

Proof (sketch). The proof follows a similar sketch as for the strict core and enemies aversion (i.e., a fulcrum-agent between MinMaxClique, inhibitors, circuit games and Example 3). However, Remark 1 does not hold with friends appreciation. However, we are still able to model gadget games and inhibitors. Here is the main idea for a construction: each agent can be forced to choose between two coalitions where her number of friends is the same but the number of enemies differ. Thus, she will prefer to be in the coalition with the lowest number of enemies if and only if it is available.

Although these results show the extreme intractability of the strict core under friends appreciation, the complexitylandscape changes radically to easiness for the core:

Theorem 9. Given an $H G / F$, (1) the existence of a core-stable coalition structure is guaranteed, and (2) it can be computed in polynomial-time as the strongly connected components of graph $G_{F}=\left(N, A_{F}\right)$.

Proof. The idea of using strongly connected components is similar to a previous result with only friends and enemies [Dimitrov et al., 2006], which can be computed in time $O\left(n^{2}\right)$ [Tarjan, 1972]. We extend it to neutrals, with a much shorter proof. Let $\pi=\left\{C_{1}, \ldots, C_{m}\right\}$ be the coalition structure corresponding to a decomposition of graph $G_{F}=\left(N, A_{F}\right)$ into strongly connected components, and let us show that it is strict core stable. Since the condensation graph, where each $C_{j}$ is contracted into one vertex, is a directed acyclic graph, it has at least one sink, which we call $C_{s}$. No subset $Y \subseteq C_{s}$ can be part of any blocking coalition $X \supset Y$, since (if $Y \subset C_{s}$ ) at least one would loose friends or (if $Y=C_{s}$ ) one may gain enemies. By repeating this argument on the condensation graph from which $C_{s}$ was removed, we obtain that no agent can be in a blocking coalition.

\section{Conclusion}

We studied the computational complexity of coalitional stability in hedonic games under enemies aversion and friends appreciation, by introducing neutral agents. It was known that without neutral agents, coalitional stability is just NPhard, while in the very general ASHGs it is $\mathrm{NP}^{\mathrm{NP}}$-complete, hence a computationally extremely hard requirement. Between these two models, to the best of our knowledge, our models are among the simplest cases of extreme intractability for coalitional stability in hedonic games. An interesting prospect is to explore assumptions that make verification tractable, in order to bring the existence problem to class NP. Also, we did not extend our results to if friend/enemy relations are symmetric. Furthermore, since very few algorithms and methods have been proposed to achieve coalitional stability, numerical experiments could be a good challenge.

\section{Acknowledgements}

This work was partially supported by JSPS KAKENHI Grant Number 17H00761 and 15H02751, and JST Strategic International Collaborative Research Program, SICORP. We are grateful to Takamasa Suzuki for his presence. 


\section{References}

[Aziz and Brandl, 2012] Haris Aziz and Florian Brandl. Existence of stability in hedonic coalition formation games. In Proceedings of the 11th International Conference on Autonomous Agents and Multiagent Systems (AAMAS2012), volume 2, pages 763-770, 2012.

[Aziz et al., 2014] Haris Aziz, Felix Brandt, and Paul Harrenstein. Fractional hedonic games. In Proceedings of the 13th International Conference on Autonomous Agents and Multi-agent Systems (AAMAS-2014), pages 5-12, 2014.

[Aziz et al., 2016] Haris Aziz, Paul Harrenstein, Jérôme Lang, and Michael Wooldridge. Boolean hedonic games. In Proceedings of the 15th International Conference on the Principles of Knowledge Representation and Reasoning (KR-2016), pages 166-175, 2016.

[Ballester, 2004] Coralio Ballester. NP-completeness in hedonic games. Games and Economic Behavior, 49(1):1-30, 2004.

[Dimitrov et al., 2006] Dinko Dimitrov, Peter Borm, Ruud Hendrickx, and Shao Chin Sung. Simple priorities and core stability in hedonic games. Social Choice and Welfare, 26(2):421-433, 2006.

[Elkind and Wooldridge, 2009] Edith Elkind and Michael Wooldridge. Hedonic coalition nets. In Proceedings of the 8th International Conference on Autonomous Agents and Multi-agent Systems (AAMAS-2009), pages 417-424, 2009.

[Ko and Lin, 1995] Ker-I Ko and Chih-Long Lin. On the complexity of min-max optimization problems and their approximation. Nonconvex optimization and its applications, 4:219-240, 1995.

[Lang et al., 2015] Jérôme Lang, Anja Rey, Jörg Rothe, Hilmar Schadrack, and Lena Schend. Representing and solving hedonic games with ordinal preferences and thresholds. In Proceedings of the 14th International Conference on Autonomous Agents and Multiagent Systems (AAMAS-2015), pages 1229-1237, 2015.

[Peters and Elkind, 2015] Dominik Peters and Edith Elkind. Simple causes of complexity in hedonic games. In Proceedings of the 24th International Joint Conference on Artificial Intelligence (IJCAI-2015), pages 617-623, 2015.

[Peters, 2015] Dominik Peters. $\Sigma_{2}^{p}$-complete problems on hedonic games. arXiv preprint arXiv:1509.02333, 2015.

[Peters, 2016] Dominik Peters. Graphical hedonic games of bounded treewidth. In Proceedings of the 30th AAAI Conference on Artificial Intelligence (AAAI-2016), pages 586593, 2016.

[Rey et al., 2016] Anja Rey, Jörg Rothe, Hilmar Schadrack, and Lena Schend. Toward the complexity of the existence of wonderfully stable partitions and strictly core stable coalition structures in enemy-oriented hedonic games. Annals of mathematics and artificial intelligence, 77(34):317-333, 2016.
[Sung and Dimitrov, 2007] Shao Chin Sung and Dinko Dimitrov. On core membership testing for hedonic coalition formation games. Operations Research Letters, 35(2):155-158, 2007.

[Tarjan, 1972] Robert Tarjan. Depth-first search and linear graph algorithms. Society for Industrial and Applied Mathematics Journal on Computing, 1(2):146-160, 1972.

[Woeginger, 2013] Gerhard J. Woeginger. A hardness result for core stability in additive hedonic games. Mathematical Social Sciences, 65(2):101-104, 2013. 